\title{
Youth services: the need to integrate mental health, physical health and social care
}

\author{
Commentary on Malla et al.: From early intervention in psychosis to youth mental \\ health reform: a review of the evolution and transformation of mental health services for \\ young people
}

\author{
Alison R. Yung ${ }^{1,2}$ (D) \\ Received: 2 February 2016/ Accepted: 20 February 2016/Published online: 18 March 2016 \\ (c) Springer-Verlag Berlin Heidelberg 2016
}

\begin{abstract}
Mental distress and mental health disorders are common in young people. Indeed, over $75 \%$ of mental disorders begin before the age of 25 years. Long delays in seeking help for illnesses are common, initial intervention is often ineffective and young people are at risk of disengaging with treatment, particularly when they are expected to move from child and adolescent treating teams to adult services. All of these factors mean that young people are vulnerable to prolonged mental ill-health and its consequences, including educational failure, unemployment, social disengagement and deprivation, and development of further mental health problems including substance misuse. Malla et al. present different service models that attempt to address these issues. Additionally, there needs to be a focus on physical health and social care as these are intertwined with mental health.
\end{abstract}

Mental distress and mental health disorders are common in young people. For example, the National Comorbidity Survey in the US reported a 12-month prevalence of mental disorders in 13-17-year-olds of over $40 \%$ [1]. These illnesses, mostly anxiety and mood disorders, tend to persist and indeed most adult mental health disorders begin in childhood or adolescence [1].

This comment refers to the article available at doi:10.1007/s00127015-1165-4.

Alison R. Yung

alison.yung@manchester.ac.uk

1 Institute of Brain Behaviour and Mental Health, University of Manchester, Manchester, UK

2 Greater Manchester West NHS Mental Health Foundation Trust, Manchester, UK
Young people tend to avoid seeking professional treatment for their mental health problems, with beliefs that it would go away, shame and embarrassment about both symptoms and the need to ask for help, fear of discrimination and concerns about confidentiality [2] all cited as reasons for this. As Malla et al. [3] report in their review, less than $30 \%$ of young people seek help for mental disorders. Long delays of several years are typical, especially for those with anxiety, mood and substance use disorders and for those with early age of onset [4].

Even after initial help-seeking there is still a risk that young people will disengage with services, and they are particularly vulnerable to this when they are expected to move from child and adolescent treating teams to adult services [5]. From attending a familiar service where one is known, the young person must move to an unfamiliar service where one is expected to behave like an adult. Thus those most at risk of development of new and secondary disorders, at a stage of their life with multiple societal and personal challenges, are presented with disjointed and often confusing array of services [3].

All of these factors mean that young people are vulnerable to prolonged mental ill-health and its consequences, including educational failure, unemployment, social disengagement and deprivation, risk of suicide and physical ill health, and the development of further mental health problems including substance misuse [6].

Clearly reform of the health system is required. Services need to respond so that young people with mental disorders can be identified early and provided with age-appropriate care that extends from early adolescence to young adulthood. Some headway has made and Malla et al. [3] review the developments in youth mental health in four countries: Australia, Ireland, the UK and Canada. 
The Australian model is one of enhanced primary care for 12-25-year-olds, with general practitioners co-located or networked with mental health workers, drug and alcohol services and educational and vocational specialists. The model (called "headspace") has been disseminated across much of Australia, with 55 sites, all adapted to local needs. "headspace" sites have proved popular with young people, with over 21,000 individuals presenting to them in the 6 month period between 1 January and 30 June 2013 [7]. Most had mild to moderate or subthreshold symptoms, but with high levels of self-reported psychological distress. The majority were functioning at least moderately well and the proportion of 18-25-year-olds engaged in education, employment or training was similar to that of the general population. A minority of headspace attendees had psychiatric diagnoses and about $6 \%$ had serious and ongoing mental disorders.

Thus "headspace" services need to balance the principle of early intervention to prevent functional deterioration and onset of illness with managing already disabled young people with chronic mental disorders. A related tension is whether the services should be targeting youth who are disengaged with employment, education and training, and others at highest risk of poor outcomes, such as young people in care, homeless youth, and those with special needs [8], or remain as walk-in centres for those with high distress but who are still functioning reasonably well. Malla et al. propose some possible solutions. One is 'stepped care', in which management is based on stage of illness. Thus initial treatments are more 'benign' and nonspecific, such as supportive psychotherapy, while treatments for later stages are more 'toxic' but specific, such as anti-depressants for depression and antipsychotics for psychotic disorders. A second solution proposed is "enhanced headspace centres" which are youth early psychosis services. However these would only cater for young people with psychotic illnesses and not those with enduring mood, anxiety, substance use or personality disorders. The issue of reaching marginalized youth has not yet been resolved, as the authors acknowledge [3].

In Canada, following extensive consultation with relevant stakeholders and a competitive process to develop a model, 12 reformed youth services are being established across the country. The services differ according to local needs and culture, and there is a focus on engaging potentially disconnected young people, including those from areas of social deprivation, homeless youth and indigenous groups. The focus is on emerging and established mental disorders.

In Ireland, youth mental health services similar to the headspace model have been established. These "Jigsaw" sites (ten across the country at present) are primary care services for young people aged 12-25 and are linked with an on-line youth mental health organization, ReachOut. com.

In the UK, a similar young people's mental health service is being established in Birmingham and is due to open in April 2016. In contrast to headspace and Jigsaw, the Birmingham service is designed for 0-25-year-olds. Unfortunately, it seems that currently this is the only dedicated youth mental health service planned for the UK. However, a number of policy statements have been made in England that include promotion of resilience and prevention as well as a focus on young people transitioning from child and adolescent services to adult services and ensuring that vulnerable young people are able to access mental health care. It is hoped that this momentum, adapting the experience in Australia, Ireland and Canada and evaluating the Birmingham site will soon translate into the establishment other youth services across the country. Clearly outcome data including economic evaluation of the existing and developing services will be of value when setting up new ones.

Malla et al. summarise some of the main problems in relation to youth mental health: high rates of untreated illnesses, delays in seeking professional help, treatments that may not be age appropriate and the requirement to transition from adolescent to adult mental health services. They also touch on the need integrate health and social care and note that the headspace services aim to address issues of employment, education, housing and justice. Additionally, helping young people with debt and accessing financial support are important given the association between social deprivation and mental illness. Other social care issues such as childcare and practical assistance for teenage parents, help with immigration issues for asylum seekers and family support should all be addressed.

Another area not discussed in the review is the physical health of young people. Young people with mental health problems have high rates of unhealthy behaviours, including substance use such as nicotine, alcohol and other substances, poor diet, and low levels of physical activity [9]. Youth mental health services can play a part in early intervention to promote healthy living, including maintenance of healthy body weight, smoking cessation, encouraging physical activity and good nutrition [9].

Young people with chronic physical diseases also face a difficult time when transitioning from child and adolescent to adult services. Non-adherence to treatment, for example for diabetes, asthma, renal failure and so on, can result in catastrophic deterioration in health and increased presentations to Emergency Departments. Young people presenting for the first time with a chronic physical health condition are at risk of poor engagement with adult health services. As psychological distress and mental disorders are frequently present in young people such chronic 
physical conditions, availability of comprehensive youthfriendly services and multidisciplinary care is important.

Malla et al. also note a growing resource for managing health problems that may be particularly attractive for young people: e-health. The Australian, Canadian and Irish models include online support services. e-health and $\mathrm{m}$-health are growth areas in many areas of medicine, and might be particularly attractive to young people, who have high rates of ownership and use of mobile phones and other electronic devices. This technology can be used in a variety ways, from simple appointment and medication reminders to delivering interventions such as online cognitive therapy, to mental health tracking and self-management strategies. Young people are willing to use such technology in the care of their mental health problems [10]. Future services developments should consider integration of these innovative methods with traditional face to face management.

Malla et al. [3] give an excellent overview of some of the sentinel work occurring in youth mental health and some recent reforms in service systems. Further work is needed. We need to build the evidence base for the acceptability, efficacy and cost-effectiveness of specialised youth services. Advocacy, involving young people and their families, may underline the importance of reforming services. Clearly funding and support from policy makers is needed.

Meanwhile, even in places where specific young people's services are absent, issues relevant to their specific needs can be addressed. All healthcare professionals who are likely to come into contact with adolescents and young adults should be aware of their developmental needs, the possibility of emerging mental health disorders and how to refer to treating teams. The potential for improving the health of young people and maximising their chances of healthy and productive lives lies with us now.

\section{Compliance with ethical standards}

Conflict of interest The corresponding author states that there is no conflict of interest.

\section{References}

1. Kessler R, Avenevoli S, Costello EJ, Georgiades K, Green J, Gruber $M$ et al (2012) Prevalence, persistence, and sociodemographic correlates of DSM-IV disorders in the National Comorbidity Survey Replication Adolescent Supplement. Arch Gen Psychiatry 69:372-380

2. Clement S, Schauman O, Graham T, Maggioni F, Evans-Lacko S, Bezborodovs $\mathrm{N}$ et al (2015) What is the impact of mental healthrelated stigma on help-seeking? A systematic review of quantitative and qualitative studies. Psychol Med 45:11-27

3. Malla A, Iyer S, McGorry P, Cannon M, Coughlan H, Singh S et al (2015) From early intervention in psychosis to youth mental health reform: a review of the evolution and transformation of mental health services for young people. Soc Psychiatry Psychiatr Epidemiol. doi:10.1007/s00127-015-1165-4 (this issue)

4. Wang PS, Berglund P, Olfson M, Pincus HA, Wells KB, Kessler RC (2005) Failure and delay in initial treatment contact after first onset of mental disorders in the National Comorbidity Survey Replication. Arch Gen Psychiatry 62:603-613

5. Singh S, Tuomainen H (2015) Transition from child to adult mental health services: needs, barriers, experiences and new models of care. World Psychiatry 14:358-361

6. Richards M, Abbott R (2009) Childhood mental health and adult life chances in post-war Britain: insights from three national birth cohort studies. http://www.rcpsych.ac.uk/pdf/life_chances_ summary2009

7. Rickwood DJ, Telford NR, Parker AG, Tanti CJ, McGorry PD (2014) Headspace-Australia's innovation in youth mental health: who are the clients and why are they presenting. Med J Aust 200:1-4

8. Deaprtment of Health (2011) No health without mental health: a cross government mental health outcomes strategy for people of all ages. Department of Health, London

9. Carney R, Cotter J, Bradshaw T, Firth J, Yung AR (2015) Cardiometabolic risk factors in young people at ultra-high risk for psychosis: a systematic review and meta-analysis. Schizophr Res. doi:10.1016/j.schres.2016.01.010

10. Lal S, Dell'Elce J, Tucci N, Fuhrer R, Tamblyn R, Malla A (2015) Preferences of young adults with first-episode psychosis for receiving specialized mental health services using technology: a survey study. JMIR Ment Health 2(2):e18. doi:10.2196/mental. 4400 